[0212-7199 (2006) 23: 8; pp 361-368] ANALES DE MEDICINA INTERNA Copyright $\odot 2006$ ARAN EDICIONES, S.L.

AN. MED. INTERNA (Madrid) Vol. 23, N. ${ }^{\circ} 8$, pp. 361-368, 2006

\section{Patrón de cumplimiento terapéutico de antihipertensivos y/o hipolipemiantes en pacientes hipertensos y/o dislipémicos en Atención Primaria}

\author{
A. SICRAS MAINAR, J. FERNÁNDEZ DE BOBADILLA' J. REJAS GUTIÉRREZ1', \\ M. GARCÍA VARGAS ${ }^{2}$
}

Servicios Asistenciales. Badalona. Investigación de Resultados en Salud y Farmacoeconomía. Unidad Médica. Pfizer I+D+i. Madrid. ${ }^{2}$ Euroclin Institute. Madrid
ANTIHYPERTENSIVE AND/OR LIPID-LOWERING TREATMENTS PATTERN OF COMPLIANCE IN HYPERTENSE AND/OR DYSLIPEMIC PATIENTS IN PRIMARY CARE

\section{RESUMEN}

Fundamento y objetivo: La falta de cumplimiento es uno de los principales factores responsables en las diferencias entre los resultados de los ensayos clínicos y la efectividad real encontrada en la práctica clínica diaria, y contribuye a un control insuficiente de los factores de riesgo cardiovascular (FRCV). Existe poco conocimiento de los factores que contribuyen a la falta de cumplimiento y en España los datos son escasos: Los objetivos de este estudio son: a) determinar de forma indirecta el grado de cumplimiento, en sujetos con hipetensión y/o dislipidemia; y b) estudiar qué factores se asocian con el cumplimiento.

Material y método: Se seleccionaron 9.001 pacientes hipertensos y/o dislipémicos adscritos a cuatro centros de atención primaria de Cataluña: a) se comparó el grado de cumplimiento entre los pacientes dislipémicos sin hipertensión (DL sin HTA), hipertensos con dislipemia (HTA con DL) e hipertensos sin dislipemia (HTA sin DL); y b) se construyó un modelo multivariante de regresión múltiple escalonado descriptivo, para explicar la variable cumplimiento terapéutico.

Resultados: 1. El cumplimiento fue 79,0\% en los DL sin HTA, significativamente menor que en los HTA DL: $81,2 \%$ ( $p<0,0001)$ y que en los HTA sin DL: $82,4 \%(\mathrm{p}<0,0001)$. También hubo diferencias significativas entre estos dos últimos grupos $(\mathrm{p}=0,001)$.

2. Las variables explicativas de un mejor cumplimiento en el análisis multivariante fueron: a) inactividad laboral $(\mathrm{p}=0,000)$; $\mathrm{b})$ relacionados con el médico que trata al sujeto $(\mathrm{p}=0,000)$ y la intensidad del seguimiento $(\mathrm{p}=0,04) ; \mathrm{y}$ ) el tipo de fármaco $(\mathrm{p}=0,000)$; el precio (a mayor precio mayor cumplimiento) $(\mathrm{p}=0,006)$ y el número de principios activos utilizados (a menor número, mayor cumplimiento) $(\mathrm{p}=0,019)$.

Conclusiones: 1. Los pacientes dislipémicos muestran un peor cumplimiento que los hipertensos y la dislipemia es un FRCV que empeora el cumplimiento global en los pacientes hipertensos.

2. Las características del sujeto, la actitud del médico, el grado de seguimiento, el tipo de fármaco empleado y la simplicidad del tratamiento se asocian al cumplimiento terapéutico.

PALABRAS CLAVE: Cumplimiento. Factores de riesgo. Hipertensión. Dislipemia.

\section{ABSTRACT}

Background and objective: Lack of compliance is a major factor responsible for the differences between clinical trial results and real effectiveness in daily medical practice, contributing to an insufficient control of the cardiovascular risk factors (CVRF). Knowledge of the factors contributing to lack of compliance is limited, and in Spain data are scarce. The objectives of this study are: a) to indirectly determine the level of compliance among patients with hypertension and/or dyslipidemia; and b) to determine factors associated with compliance.

Material and method: 9,001 hypertensive and/or dyslipidemic patients from four primary care centres in Catalonia were enrolled in Disease Management programmes during the previous four years. Compliance was estimated by the relationship between the amount of dispensed and prescribed pills: a) the levels of compliance of dyslipidemic patients without hypertension (DL-non HT), hypertensive patients with dyslipidemia $(H T+D L)$ and hypertensives without dyslipidemia (HT-non $D L)$ were compared; and b) an stepwise, multivariate, descriptive; multiple regression model was designed in order to explain compliance.

Results: 1. Compliance was $79 \%$ in DL-non HT, significantly lower than in $H T+D L(81.2 \%, p=0.000)$ and in $H T$-non DL $(82.4 \%, p=0.000)$. There were also statistically significant differences between these last two groups $(p=0.001)$.

2. Explanatory variables of a better compliance in the multivariate analysis were: a) patient related factors: labour inactivity $(p=0.000)$; b) management related factors: specific doctor $(p=0.000)$ and intensity of follow-up $(p=0.04)$; and $c)$ drug related factors: the drug group $(p<$ 0.0001 ); the drug price (the higher price determines higher compliance, $p=0.006$ ) and the number of active principles used (the higher number determines lower compliance, $p=0.019$ ).

Conclusions: 1. Dyslipidemic patients show a worse compliance than hypertensive patients, and dyslipidemia worsened global compliance in hypertensive patients.

2. Patient characteristics, doctor attitude, follow-up intensity, drug group and simplicity of treatment are related to compliance in daily medical practice.

KEY WORDS: Compliance. Risk factors. Hipertensión. Dislipemia.

Sicras Mainar A, Fernández de Bobadilla J, Rejas Gutiérrez J, García Vargas M. Patrón de cumplimiento terapéutico de antihipertensivos y/o hipolipemiantes en pacientes hipertensos y/o dislipémicos en Atención Primaria. An Med Interna (Madrid) 2006; 23: 361-368. Resultados preliminares de este trabajo han sido presentados en el $8^{\text {th }}$ Annual European Congress of the International Society for Pharmacoeconomics and Out-
comes Research (ISPOR), Florencia, Noviembre 2005. Este estudio ha sido financiado por Pfizer, S.A.

Trabajo aceptado: 13 de marzo de 2006

Correspondencia: Jaime Fernández de Bobadilla. Dpto. IRS y Farmacoeconomía. Unidad Médica. Pfizer S. A. Avda. de Europa, 20-B. Parque Empresarial La Moraleja. 28108 Alcobendas (Madrid). Fax: 9149097 20. e-mail: Jaime.Fernandez@pfizer.com 


\section{INTRODUCCIÓN}

La hipertensión arterial y la hipercolesterolemia constituyen, junto a la diabetes mellitus, los principales factores de riesgo modificables para el desarrollo de las enfermedades cardiovasculares de los países desarrollados (1-3). La efectividad de una intervención sanitaria no puede establecerse de una forma absoluta si no se conoce el grado de cumplimiento (adhesión, concordancia o intensidad de uso) al tratamiento farmacológico recomendado por los profesionales de la salud (4-6).

El impacto potencial del incumplimiento terapéutico en resultados de tratamiento y costes es elevado. Se ha estimado que de más de dos mil millones de prescripciones anuales realizadas en EE.UU., sólo la mitad se toman adecuadamente y muchos expertos afirman que el incumplimiento es responsable de una proporción importante del gasto sanitario. Además, solamente en el tratamiento de la enfermedad cardiaca, se considera que el incumplimiento causa 125.000 muertes evitables cada año (7). Por tanto, el incumplimiento terapéutico a largo plazo es uno de los problemas más importantes con el que nos enfrentamos en el tratamiento de las enfermedades crónicas en situación la práctica clínica; puesto que se disminuye la eficiencia sanitaria, con una falta de consecución de objetivos de tratamiento y un aumento de los costes sanitarios asociados (8-10).

Para valorar el cumplimiento terapéutico se dispone de métodos directos o indirectos de evaluación. Los métodos directos se basan en determinaciones de metabolitos o marcadores biológicos, mediante analíticas de sangre y orina, y son de difícil aplicación práctica. Entre los métodos indirectos destacan el recuento de formas farmacéuticas sobrantes, el control de la dispensación, los cuestionarios para declaraciones del paciente mediante entrevistas, los sistemas de monitorización electrónica o la revisión de registros de farmacia, siendo estos últimos los más utilizados (11).

Como causa de baja adherencia a un tratamiento crónico destaca la ausencia de síntomas, el temor a las reacciones adversas y el desconocimiento de los riesgos derivados de la propia enfermedad. Se han identificado diversas estrategias que mejoran la adherencia y, en consecuencia, los resultados terapéuticos: disminución de la complejidad del tratamiento, administración de información verbal y escrita, mejora de la comunicación médico-paciente. La combinación de varias intervenciones ha mostrado una mayor eficacia (12).

En nuestro medio la falta de cumplimiento es un problema de primera magnitud $(13,14)$. En España, la prevalencia del incumplimiento farmacológico en enfermedades crónicas oscila entre el 16-75\%, con una importante variabilidad en cuanto al método de medida (2-5). Algunos autores respaldan el valor de los programas educativos para mejorar el cumplimiento $(14,15)$. En todo caso, los datos en la práctica de la atención primaria son escasos.

El objetivo de este estudio es evaluar, el grado de cumplimiento terapéutico del tratamiento farmacológico en pacientes con hipertensión arterial leve-moderada, y/o hipercolesterolemia, en situación de práctica clínica habitual en el ámbito de la atención ambulatoria.

\section{PACIENTES Y MÉTODOS}

Se realizó un estudio observacional, longitudinal, retrospectivo y multicéntrico, con muestreo sistemático, a partir de los registros médicos de pacientes adultos seguidos en régimen ambulatorio y en situación de práctica clínica habitual. La población de estudio está formada por las personas de ambos sexos de cuatro centros reformados de atención primaria (ABS Apenins-Montigalá, ABS Morera-Pomar, ABS Montgat-Tiana y ABS Nova Lloreda), gestionados por Badalona Serveis Assistencials SA, que tienen asignados en su zona de influencia a una población urbana de unos 80.298 habitantes, de los que un 14,1\% son mayores de 64 años.

Se incluyeron en el estudio a todos los pacientes atendidos desde el 1 de octubre de 2003 al 30 de septiembre de 2004 que reunían las siguientes características: a) edad superior a 18 años; b) haber iniciado el tratamiento farmacológico 3 meses antes de la inclusión en el estudio; c) pacientes diagnosticados de hipertensión esencial y/o dislipemia entre 3 años y 12 meses antes del inicio del estudio; d) seguir de forma regular el protocolo/guía de riesgo cardiovascular establecido en los centros; e) estar en el programa de prescripciones crónicas y acudir con regularidad a las citas programadas para la obtención de las recetas médicas (con registro constatado de la dosis diaria, el intervalo de tiempo y la duración de cada tratamiento administrado); y f) haberse dispensado más de 5 envases de productos farmacéuticos durante el periodo de estudio. Fueron excluidos los pacientes que no acudieron a los centros, los desplazados o fuera de zona, los que sufrían enfermedades mentales incapacitantes, la coexistencia con la hipertensión arterial y/o la hipercolesterolemia con enfermedades mentales incapacitantes, los que tenían hipertensión arterial secundaria o maligna. Mediante estos criterios se pretendió, por una parte, evitar a los pacientes muy complejos con comorbilidad asociada y tratados con múltiples fármacos, y por otra, evitar a los pacientes incluidos muy recientemente en los cuales no se ha alcanzado una estabilidad clínica suficiente, puesto que el desfase entre la prescripción, la dispensación y la toma del medicamento podría dar lugar a una interpretación inadecuada.

El diagnóstico de hipertensión arterial leve-moderada se estableció según los criterios de la JNC-VII (16), siguiendo las indicaciones de la British Hipertensión Society para su medición (17) y los de la NCEP-ATP III (18) para la hipercolesterolemia.

El estudio se realizó en dos etapas. En la primera se seleccionaron a todos los pacientes en tratamiento farmacológico previamente establecido y en la segunda, considerada de evaluación clínica, se obtuvieron las variables clínicas y de utilización de recursos necesarias para su asociación. Finalmente se confeccionó una única base de datos, para el posterior análisis de los datos. Se incluyeron pacientes con las siguientes patologías, que definieron 3 cohortes de pacientes: a) hipertensos esenciales no dislipémicos en tratamiento con antihipertensivos; b) dislipémicos no hipertensos en tratamiento con estatinas; y c) hipertensos esenciales dislipémicos en tratamiento con estatinas y antihipertensivos de forma simultánea.

Se obtuvo información de la prescripción farmacológica de medicamentos y efectos y accesorios, emitida en las historias clínicas informatizadas (OMI-AP) según las recomendaciones realizadas por el facultativo, y de la dispensación obtenida a partir de las recetas retiradas de la oficina de farmacia, según el aplicativo de seguimiento de la prescripción farmacéutica (SPF) del CatSalut. En los dos apartados (prescripción/dispensación) se han seleccionado como grupos terapéuticos (19) los siguientes antihipertensivos, agrupados en: diuréticos $(\mathrm{C} 03 \mathrm{~A}, \mathrm{C} 03 \mathrm{~B}$, $\mathrm{C} 03 \mathrm{C}$ ), calcioantagonistas (C01D2 y amlodipino solo), betabloqueantes beta-adrenérgicos (C07A), betabloqueantes alfa-adre- 
nérgicos $(\mathrm{C} 02 \mathrm{~A}, \mathrm{C} 02 \mathrm{C})$ y hipotensores sobre el sistema renina angiotensina $(\mathrm{C} 02 \mathrm{E}$; con diferenciación específica entre los inhibidores de la enzima convertasa y de los antagonistas de la angiotensina II) y diferentes asociaciones de fármacos (C03D, C02F, C07B); y como hipolipemiantes (B04A) los principios activos: lovastatina, simvastatina, pravastatina, fluvastatina, atorvastatina y otros hipolipemiantes como fibratos. Además, se constató el número de grupos terapéuticos o de fármacos consumidos (principios activos) durante el periodo de seguimiento.

El cumplimiento se definió como el grado de coincidencia o concordancia del comportamiento de un paciente (en relación al consumo de la medicación, el seguimiento de una dieta o modificaciones que ha de realizar en su estilo de vida) con las recomendaciones médicas o de los profesionales de la salud que lo atienden. La magnitud del incumplimiento terapéutico se calculó en cada paciente y para los grupos terapéuticos seleccionados, mediante el método indirecto modificado del recuento de comprimidos. Una vez identificada la fecha de incorporación a las bases de datos, y hasta la última visita disponible, se cuantificó el tiempo transcurrido y las prescripciones o dispensaciones realizadas. Se calculó el porcentaje de cumplimiento del período como el número total de comprimidos dispensados entre el número total de comprimidos recomendados o prescritos, asumiendo que la dispensación del fármaco (adquisición de la medicación en la farmacia), no supone su consumo o toma real, pero se asocia estrechamente con ésta. Se analizaron individualmente para antihipertensivos e hipolipemiantes y de manera conjunta para aquellos pacientes que comparten ambos tratamientos. Se consideraron buenos cumplidores los pacientes que presentaron valores superiores al $80 \%$.

Se obtuvieron las variables demográficas y generales: edad, sexo, actividad laboral (situación en la seguridad social; activo-pensionista), unidad médica asignada, evolución en meses de la hipertensión arterial leve-moderada y/o de la hipercolesterolemia (dicotómica: hasta los dos, o más de dos años desde la fecha del diagnóstico inicial).

Los diagnósticos clínicos o comorbilidades asociadas a la hipertensión arterial leve-moderada y/o a la hipercolesterolemia se han obtenido a partir de la Clasificación Internacional de la Atención Primaria (CIAP-2) (20). Los episodios atendidos, no repetidos, en población historiada seleccionados fueron: población diana (hipertensión arterial leve-moderada, K86; hipercolesterolemia, T93 parcial), diabetes mellitus (T90: todos los tipos), fumador (P17), obesidad (T82), cardiopatía isquémica (K74: isquemia cardiaca con angina, K75: infarto agudo de miocardio, K76: isquemia coronaria), accidente cerebrovascular (K90, K91: isquemia cerebral transitoria), enfermedad arterial periférica (K92: claudicación intermitente, síndrome de Raynaud, estenosis o embolismo arterial), insuficiencia cardiaca congestiva (K77), enfermedad pulmonar obstructiva crónica (R95: obstrucción crónica del flujo aéreo), trastornos depresivos (P76: todos los tipos), insuficiencia renal (U99), cirrosis hepática (D97) y hipertrofia benigna de próstata (Y87). Además se cuantificó el número de factores de riesgo cardiovascular.

Cómo parámetros clínicos se obtuvo: el índice de riesgo cardiovascular (cálculo adaptado de Framingham para la atención primaria), índice de masa corporal (IMC, $\mathrm{kg} / \mathrm{m}^{2}$ ), presión arterial sistólica (PAS, $\mathrm{mmHg}$ ) y diastólica (PAD, mmHg), colesterol total $(\mathrm{mg} / \mathrm{dL})$, fracción de colesterol-lipoproteínas de baja densidad (cLDL, obtenida según la fórmula de Friedewald en mg/dL) (21) y fracción de colesterol-lipoproteínas de alta densidad (cHDL) en $\mathrm{mg} / \mathrm{dL}$.
Teniendo en cuenta que, para cuantificar el adecuado grado de control de un paciente, es preciso tener en cuenta otras variables relacionadas con sus diferentes factores de riesgo cardiovascular, se obtuvieron las variables necesarias para definir el nivel de control de acuerdo con los criterios definidos, para el control de la dislipemia del ATP-III, y de la hipertensión arterial del JNC-VII. Posteriormente se construyó la variable control/no control, dependiendo de los criterios especificados en estas recomendaciones. Se consideraron como buenos objetivos de seguimiento o control en presión arterial (TAS/TAD; < 140/90 mmHg o $<130 / 80$ en diabéticos) y cLDL $(<190,<160$ o $<130$ $\mathrm{mg} / \mathrm{dL}$ dependiendo de la presencia de factores de riesgo y enfermedad cardiovascular o diabetes). Además, se realizaron otras agrupaciones de monitorización de resultados aceptables u óptimos según rangos de la NCEP-ATP III (18) como control de los objetivos terapéuticos. En todos los casos (exploración física o determinaciones bioquímicas) se consideró la última medida obtenida durante el período de estudio.

Los recursos sanitarios consumidos obtenidos a partir de los registros propios de cada centro (historias clínicas, órdenes médicas, agendas programadas y emisión de prescripciones) fueron: número de entradas efectuadas en el protocolo de riesgo cardiovascular, las derivaciones realizadas a los especialistas de referencia, las visitas o citas realizadas en el centro y el coste total del tratamiento.

Los datos se obtuvieron de forma informatizada, respetándose la confidencialidad de los registros marcada por la ley. En la metodología de cálculo, la fuente de información la constituyen las diferentes bases de datos de las historias clínicas. Se ha efectuado una validación y depuración de la información introducida a partir de documentos internos (cuadro de mando de la organización) y de rangos de variables, en especial con los datos de prescripción y dispensación farmacéutica; y se eliminaron los datos incoherentes. La matriz de datos final y el cruce de las variables se han realizado a partir del desarrollo de una aplicación propia creada con el soporte de Microsoft Access ${ }^{\circledR}$.

Se ha efectuado un análisis estadístico descriptivo univariante con valores de media y desviación estándar (DE). Se comprobó la normalidad de la distribución con la prueba de KolmogorovSmirnov y se aplicaron intervalos de confianza al 95\% (IC). La relación entre las variables independientes con la dependiente (cumplimiento terapéutico) se estudió con la pruebas de la t de Student (U de Mann Whitney si no cumplían las condiciones de aplicación), ANOVA, la Chi cuadrado y la correlación binaria de Pearson. Se efectuó un análisis de regresión logística múltiple, con procedimiento paso a paso hacia delante para la corrección del modelo, tomando como variable dependiente el cumplimiento terapeútico. Las variables se incluyeron en función de la significación de los resultados en el análisis bivariante y de su significado clínico. Se utilizó el programa SPSS para Windows, estableciendo una significación estadística para valores de $\mathrm{p}<0,05$.

\section{RESULTADOS}

De una selección inicial de 67.806 pacientes mayores de 18 años asignados a los centros, 14.175 cumplían criterios de hipertensión esencial y/o hipercolesterolemia; de ellos, 5.862 $(41,4 \%)$ con hipertensión arterial sin hipercolesterolemia (HTA-no DL), 5.275 (37,2\%) con hipercolesterolemia sin hipertensión arterial (DL-no HTA) y $3.038(21,4 \%)$ con hipertensión arterial e hipercolesterolemia (HTA-DL). Una vez 
descartados los pacientes que no estaban tomando tratamiento farmacológico y los que cumplían con los criterios de exclusión, la población final de estudio fue de 9.001 pacientes.

Las características generales de la serie estudiada se detallan en la tabla I. Todos ellos presentaban diagnóstico de HTA y/o hipercolesterolemia con más de dos meses de anterioridad al inicio del estudio. Casi la mitad de los pacientes, el 48,1\%

\section{TABLA I}

\section{CARACTERÍSTICAS GENERALES DE LA SERIE ESTUDIADA} $(\mathrm{N}=9.001)$

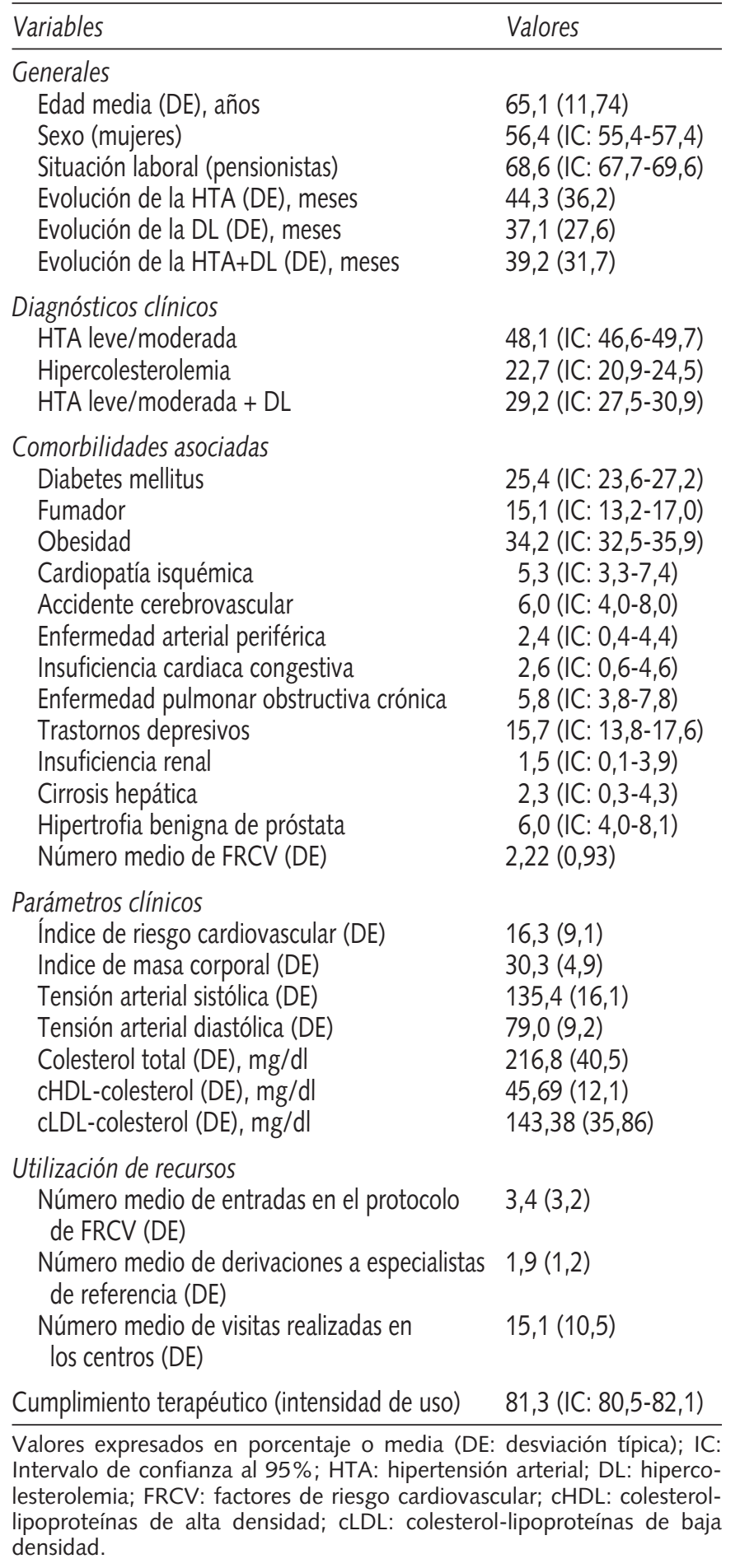

eran hipertensos sin dislipemia, el 22,7\% únicamente dislipémicos, y el 29,2\% eran hipertensos e hipercolesterolémicos. La duración de HTA y DL en más de la mitad de los casos había sido de 3 años, y en el 25\% unos 5 años. Como valores promedio de los parámetros clínicos de TAD, cHDL y de cLDL, cabe destacar que un $50 \%$ de los pacientes presentaba valores de TAD por encima de los $80 \mathrm{mmHg}$, menos de la mitad de la muestra presentaban valores de $\mathrm{cHDL}>40 \mathrm{mg} / \mathrm{dl}$ y un $25 \%$ presentaba valores de cLDL por encima de 165 $\mathrm{mg} / \mathrm{dl}$. Más de la mitad de los pacientes tenía un colesterol total mayor de $214 \mathrm{mg} / \mathrm{dl}$. Los pacientes presentaban en promedio 2,2 (DE: 0,9) factores de riesgo cardiovascular, aunque el $62,9 \%$ tenían 3 o más factores de riesgo cardiovascular (Tabla II). El porcentaje de eventos cardiovasculares fue del $17,7 \%$. Además, el número medio de visitas al médico de atención primaria fue de 15,1 (DE: 10,5); el 25\% de los pacientes acudió más de 20 veces a su médico en el periodo de estudio.

\begin{tabular}{cc} 
TABLA II \\
DISTRIBUCIÓN DEL NÚMERO DE FACTORES DE RIESGO \\
CARDIOVASCULAR (FRCV) \\
\hline Número de FRCV & Proporción de sujetos (\%) \\
\hline 1 & 7,9 \\
2 & 29,2 \\
3 & 43,2 \\
4 & 17,1 \\
5 & 2,1 \\
\hline
\end{tabular}

Del total de fármacos prescritos, un 18,2\% correspondían hipolipemiantes y un 56,9\% a terapia antihipertensiva (Tabla III). El 56,0\% (IC: 54,9-57,0) de los pacientes recibía tratamiento con dos o más fármacos. El coste medio por tratamiento/año se sitúa en $8.379,74 €$ (DE: $14.912,94)$. Un $10 \%$ de los tratamientos supusieron un coste en precio venta al público superior a $21.000 €$.

\section{TABLA III}

CARACTERÍSTICAS DEL TRATAMIENTO FARMACOLÓGICO

\begin{tabular}{lcc}
\hline Tipos de fármacos & $n$ & Porcentaje \\
\hline Hipolipemiantes & 1.637 & 18,2 \\
Lovastatina & 204 & 2,3 \\
Simvastatina & 669 & 7,4 \\
Pravastatina & 81 & 0,9 \\
Atorvastatina & 348 & 3,9 \\
Fluvastatina & 65 & 0,7 \\
Otros hipolipemiantes & 270 & 3,0 \\
Diuréticos & 647 & 7,2 \\
Calcio antagonistas & 553 & 6,1 \\
Beta bloqueantes & 604 & 6,7 \\
Alfa bloqueantes & 161 & 1,8 \\
IECA & 2.201 & 24,4 \\
ARA II & 955 & 10,6 \\
Asociaciones & 2.242 & 24,9 \\
\hline
\end{tabular}

IECA: inhibidores de la enzima convertidora de angiotensina; ARA II: antagonistas del receptor de angiotensina II. 
El cumplimiento terapéutico medido a través de intensidad de uso fue del 82,4\% para los HTA-no DL, del 79,0\% para los DL-no HTA y del 81,2\% para los hipertensos dislipémicos HTA-DL $(F=32,846 ; p=0,000$; contrastes a posteriori de Scheffé significativos). Los sujetos con 0-1 FRCV tenían menos cumplimiento que los de 2 o más, y que los que tenían enfermedad cardiovascular o equivalente $(\mathrm{p}=0,01)$. En el análisis bivariado hubo relación significativa de la variable cumplimiento: a) para las variables demográficas edad y actividad laboral (regimen de pensionistas a la seguridad social); b) para todas las variables relacionadas con la actitud del médico y el paciente (médico, número de entradas al protocolo, centro, número de visitas); c) para muchas variables relacionadas con la comorbilidad y severidad: presencia de hipertensión, dislipemia, EPOC, hipertrofia prostática, enfermedad cardiovascular, depresión, tabaquismo, meses de duración de la hipertensión y dislipemia, número de factores de riesgo cardiovascular; d) para variables relacionadas con datos clínicos y analíticos: valor de presión arterial diastólica, valor de HDL en $\mathrm{mg} / \mathrm{dL}$, valor de LDL, valor de colesterol total, valor de presión arterial sistólica; y e) para variables relacionadas con el tratamiento: tipo de fármaco, número de principios activos, control simultáneo de la presión arterial y lípidos, control de la presión arterial y el precio (Tabla IV). No hubo asociación para las variables sexo, presencia de diabetes, presión arterial sistólica y grado de control de cLDL.

Se incluyeron inicialmente en el modelo multivariante las variables que tuvieron una significación estadística en el análisis bivariante. El índice de colinealidad fue inferior a 10, y cuando dos variables mostraron una fuerte colinealidad se eliminó la de menor capacidad predictiva en el modelo. Las variables explicativas de un mejor cumplimiento en el análisis de regresión lineal múltiple fueron, entre las demográficas: la inactividad laboral $(\mathrm{p}=0,000)$, entre las relacionadas con la actitud del médico y el paciente: el médico concreto del paciente $(\mathrm{p}=0,000)$ y la intensidad del seguimiento ( $\mathrm{p}=0,04)$; entre las analíticas: los valores de colesterol total y cHDL $(\mathrm{p}<0,0001)$; entre las relacionadas con el tratamiento farmacológico: el tipo de fármaco $(\mathrm{p}<$ 0,0001 ), el precio (a mayor precio mayor cumplimiento) ( $\mathrm{p}$ $=0,006)$ y el número de principios activos utilizados (a menor número, mayor cumplimiento) $(\mathrm{p}=0,019)$. Los resultados del análisis del análisis multivariante de regresión múltiple se muestran en la tabla V.

En relación al grado de control de los FRCV, únicamente un 27,0\% (IC: 26,2-28) de los pacientes, tenían simultáneamente controlados la tensión arterial y la cLDL; un 60,5\% (IC: 59,4-61,5) tenían adecuadamente controlada la tensión arterial. La proporción de pacientes con control
TABLA IV

RESULTADO DEL ANÁLISIS BIVARIADO EN EL CUMPLIMIENTO TERAPÉUTICO

\begin{tabular}{lc}
\hline Adhesión al tratamiento & \\
\hline Variables & Valor de $p$ \\
\hline Tipo de patología & 0,000 \\
Tipo de hipolipemiante & 0,210 \\
Edad & 0,000 \\
Sexo & 0,140 \\
Evento CV & 0,027 \\
Duración HTA y DL & 0,129 \\
Situación laboral (pensionistas) & 0,000 \\
Médico & 0,000 \\
Intensidad de seguimiento & 0,000 \\
HBP & 0,002 \\
EPOC & 0,021 \\
DM & 0,379 \\
Fumador & 0,000 \\
SD & 0,000 \\
TAD & 0,009 \\
HDL & 0,004 \\
CT & 0,000 \\
n'PA & 0,021 \\
Precio medicación & 0,000
\end{tabular}

CV: cardiovascular; EPOC: enfermedad pulmonar obstructiva crónica; HBP: hiperplasia benigna de próstata; DM: Diabetes Mellitus; SD: síndrome depresivo; TAD: tensión arterial sistólica; CT: colesterol total; PA: principios activos.

óptimo del cLDL de acuerdo a las recomendaciones del ATP III fue del $40,1 \%$ para los HTA sin DL, del $29,6 \%$ para los DL sin HTA y del $27,8 \%$ para los HTA con DL (Tabla VI). Tenían simultáneamente niveles óptimos de cLDL y presión arterial un $27,1 \%$ de los sujetos, con las siguientes proporciones en cada grupo: HTA sin DL: $30,1 \%$; DL sin HTA: 27,5\%; DL con HTA: $21,9(p=0,000)$.

$\mathrm{Si}$ consideramos que el objetivo de control para los pacientes con enfermedad coronaria o equivalente de enfermedad coronaria es de $\mathrm{cLDL}<100 \mathrm{mg} / \mathrm{dL}$; para los pacientes con 2 o más factores de riesgo menor de $130 \mathrm{mg} / \mathrm{dL}$ y para los que tienen 1 o ningún factor de riesgo de $160 \mathrm{mg} / \mathrm{dL}$, las cifras de objetivo de control en el total de la población, se alcanzarían en el $48,7 \%$ de la población del estudio, de acuerdo a las recomendaciones del ATP III.

TABLA V

RESULTADOS DEL ANÁLISIS MULTIVARIANTE DE REGRESIÓN MÚLTIPLE

\begin{tabular}{|c|c|c|c|c|c|c|c|c|c|c|c|c|c|}
\hline \multicolumn{14}{|c|}{ Adhesión al tratamiento } \\
\hline Variables & $\begin{array}{c}\text { Situación } \\
\text { laboral } \\
\text { (pensionista) }\end{array}$ & Médico & $\begin{array}{l}\text { Intensidad de } \\
\text { seguimiento }\end{array}$ & $H B P$ & $D M$ & Fumador & SD & $T A D$ & $H D L$ & $C T$ & $\begin{array}{c}\text { Tipo de } \\
\text { medicación }\end{array}$ & $n^{\circ} P A$ & $\begin{array}{c}\text { Precio } \\
\text { medicación }\end{array}$ \\
\hline Valor de $p$ & $<0,0001$ & $<0,0001$ & 0,0404 & 0,15 & 0,058 & 0,23 & 0,062 & 0,058 & 0,043 & $<0,0001$ & $<0,0001$ & 0,019 & 0,0062 \\
\hline
\end{tabular}


TABLA VI

\begin{tabular}{lccc}
\multicolumn{4}{c}{ CONTROL DE LOS FACTORES DE RIESGO } \\
CARDIOVASCULARES \\
\hline CLDL (mg/dl) & STA & DL & HTA \\
& sin DL $\%)$ & sin HTA $(\%)$ & con DL $(\%)$ \\
\hline Óptimo (<100) & $40,1 \%$ & $29,6 \%$ & $27,8 \%$ \\
Casi óptimo (100-129) & $19,2 \%$ & $19,3 \%$ & $17,6 \%$ \\
Límite alto (130-159) & $25,2 \%$ & $24,2 \%$ & $24,0 \%$ \\
Alto (160-189) & $12,7 \%$ & $15,2 \%$ & $18,4 \%$ \\
Muy alto ( $\geq 190)$ & $2,8 \%$ & $11,7 \%$ & $12,3 \%$ \\
\hline
\end{tabular}

HTA: hipertensión; DL: hipercolesterolemia.

\section{DISCUSIÓN}

La utilidad de los fármacos hipolipemiantes para reducir la morbi-mortalidad cardiaca es bien conocida. Un mal cumplimiento compromete seriamente su eficacia, causando morbimortalidad evitable, lo que provoca "una considerable carga clínica y económica para el sistema de salud" (22). Para poder mejorar el cumplimiento terapéutico, el primer paso es identificar qué factores lo condicionan o, al menos, con qué factores se asocia. Como las causas que condicionan el mayor o menor cumplimiento terapéutico pueden tener importantes variaciones dependiendo del país, tiene gran interés contar con datos locales. En general existen pocos datos sobre los factores que determinan el cumplimiento terapéutico en nuestro país (1115), esto es también verdad para el cumplimiento terapéutico en hipertensión y dislipemia. Por otro lado, en ausencia de grandes tamaños muestrales resulta difícil obtener relaciones signifcativas con la variable cumplimiento, ya que el cumplimiento terapéutico depende en gran parte de factores individuales intangibles.

Una de las mayores dificultades para medir el cumplimiento terapéutico es que todos los instrumentos de medida que se utilicen para medir el cumplimiento de forma prospectiva, tienen el problema del efecto de sentirse observado o efecto Hawthorne (12). Es decir, que siempre que un sujeto es consciente de que su cumplimiento está siendo evaluado, mejora el cumplimiento de forma que es imposible evaluar con precisión el cumplimiento real en la práctica clínica. Este efecto también tiene influencia sobre el médico, ya que su actitud se modifica al sentirse observado. Por otro lado, el cumplimiento medido en los ensayos clínicos tiene poco que ver con el de práctica clínica habitual, ya que están específicamente diseñados para maximizarlo (3). En nuestro estudio, de carácter retrospectivo, no tenemos el efecto de sentirse observado; pero sí todas las dificultades propias de este tipo de estudios, entre las que se cuentan la dificultad para conseguir datos de calidad, la ausencia frecuente de variables importantes de interés y, sobre todo, que la variable cumplimiento casi nunca está disponible. No obstante, es un tipo de diseño adecuado para evitar este efecto y puede resultar especialmente atractivo para contestar preguntas relacionadas con el cumplimiento terapéutico en situación de práctica clínica habitual. Parte de los problemas propios de un estudio retrospectivo se soslayaron, al menos parcialmente, recurriendo a datos en un entorno en el que, de rutina, la recogida de datos en la práctica clínica es de gran calidad y además se recogen específicamente muchos datos relacionados con el riesgo cardiovascular y con la atención clínica. Aunque, obviamente, es en la práctica imposible tener retrospectivamente información sobre todas las variables a las que se supone alguna relación con el cumplimiento, esta aproximación permite que los grandes grupos de factores que afectan al cumplimiento estén representados por alguna de las variables recogidas en la rutina de la práctica clínica. Tenemos de esta forma información sobre factores demográficos, relacionados con la actitud del médico y el paciente, con la comorbilidad y severidad, con datos clínicos y analíticos y con el tratamiento: tipo de fármaco.

Los pacientes dislipémicos tienen peor cumplimiento que los hipertensos y la dislipemia condiciona un peor cumplimiento en los pacientes hipertensos. Aunque no se puede deducir diréctamente de los resultados; parecería lógico pensar que medidas que asocien el tratamiento de la dislipemia a la hipertensión, podrían aumentar los niveles de cumplimiento. Un dato interesante es el que se refiere a la utilización de los fármacos en función de las características de los pacientes. Parece claro que el perfil de riesgo selecciona algún tipo concreto de fármacos. A pesar de que no fue un objetivo específico de nuestro estudio, se pudo observar que entre las diferentes estatinas, Atorvastatina se usó en pacientes con mayor riesgo cardiovascular. Un 57,4\% de los pacientes presentaban antecedentes de enfermedad cardiovascular o equivalente (diabetes) vs. un 42,9\% de los que usaron otras estatinas ( $\mathrm{p}=$ 0,000). Una proporción significativamente mayor de pacientes con Atorvastatina, alcanzaron un control óptimo de cLDL, en comparación con las otras estatinas: 52,8 vs. 46,47\% (p < $0,01)$. En la práctica clínica diaria Atorvastatina se usa en pacientes con más FRCV y peor perfil de riesgo que las otras estatinas, sin embargo, es la que logra un mayor porcentaje de pacientes dentro de objetivos de control del ATP III. Los datos de cumplimiento son altos en este estudio, un $82,4 \%$ para los HTA-no DL, de un 79,0\% para los DL-no HTA y de un 81,2\% para los hipertensos dislipémicos HTA-DL. Probablemente por dos razones ya mencionadas: atención clínica por encima del promedio, y estimación indirecta del cumplimiento a partir de la relación entre la prescripción y dispensación. El menor cumplimiento en los pacientes dislipémicos en relación con los hipertensos 79,0 vs. 82,4\% ( $\mathrm{p}<0,0001)$ es también consistente con los datos publicados en la literatura (12-15). Entre los factores que se relacionan con el cumplimiento terapéutico en algunos estudios está el sexo femenino. En un estudio las mujeres tenían mejor cumplimiento al año (80,0 vs. 77,0\%), sin embargo no hubo diferencias significativas a los 4,5 años $(47,0$ vs. 46,0\%) $(23,24)$. En nuestro estudio no hubo relación con el sexo femenino en el análisis bivariado, ni el sexo femenino fue un predictor independiente en el modelo. Esto es consistente con lo publicado en la literatura, ya que en nuestro estudio se incluyeron pacientes que ya llevaban tiempo en tratamiento.

En cuanto a las variables que mostraron un mejor cumplimiento en el análisis multivariante llama especialmente la atención que el cumplimiento fuese mayor a mayor precio. Probablemente esto es específico de países con una medicina fuertemente socializada y de enfermedades crónicas propias de pensionistas en las que el coste de adquisición del medicamento para el paciente es muy pequeño. En otros países hay datos que indican que los pacientes con enfermedades cróni- 
cas asintomáticas, como la hiperlipidemia, son más reacios a pagar una parte importante de sus ingresos por las medicinas . No obstante, en el caso de la hipertensión, es cierto que los fármacos más baratos como los diuréticos y betabloqueantes, tienen limitado el cumplimiento por el mayor número de efectos adversos, que es un factor especialmente limitante del cumplimiento terapéutico (25). Otro dato de interés es que la simplicidad del tratamiento se relaciona en el modelo con un mayor cumplimiento terapéutico. Resultan predictores independientes de un mejor cumplimiento factores relacionados con la actividad laboral, la actitud del médico y el paciente, el fármaco. Esto no significa que estos sean los únicos factores que influyen en el cumplimiento, sino que la capacidad explicativa del modelo era óptima utilizando estas variables.

Una de las limitaciones del estudio es que, aunque la población es representativa de la población española de hipertensos y dislipémicos (validez externa). No obstante, son pacientes que tienen un grado de control más alto que el del promedio de la población española: las cifras de objetivo de control de cLDL de acuerdo a las recomendaciones del ATP III (18) en el total se alcanzaron en el 48,7\% de la población del estudio., y tenían un adecuado control de la presión arterial un $60,5 \%$. Sin embargo, dadas las necesidades peculiares necesidades de este diseño retrospectivo, solo tenía sentido el estudio en un entorno en el que se recogieran datos de calidad en la práctica clínica diaria y con un seguimiento estrecho (el promedio de visitas/año fue de 15). Probablemente esto condiciona una asociación con una calidad asistencial mejor y un control global mayor que el promedio. No obstante, no hay motivos para pensar que los factores que determinan el cumplimiento terapéutico, cuya determinación era el objetivo del estudio, se vean particularmente alterados por el hecho de un mejor control global. Por otro lado, las dificultades relacionadas con la medida del cumplimiento en sí, se soslayaron midiendo un parámetro objetivo, el cociente medicación prescrita y medicación dispensada. Es cierto que esta no es una medida directa del cumplimiento, si no de la intensidad de uso; pero aunque la dispensación no garantiza que el fármaco se tome, este método para estimar el cumplimiento, cuando está disponible es muy valioso, porque la mayoría de los pacientes no intentan engañar al farmacéutico a propósito (26), por tanto, existe un sólida asociación entre la dispensa- ción y la toma real del medicamento. Pese a la diversidad de variables predictoras que influyen sobre el cumplimiento terapéutico, sólo una pequeña parte de la variable cumplimiento pudo ser explicada con el modelo. Esto es consistente con la naturaleza del propio cumplimiento, afectado por infinidad de factores de orden psicológico, emocional y social que son muy difíciles de cuantificar en un estudio. Este hecho explica también la dificultad para encontrar factores que influyan en el cumplimiento terapéutico con tamaños muestrales pequeños. Además, existen limitaciones propias del método de valoración del cumplimiento, ya que la dispensación no significa necesariamente que el paciente se tome la medicación. Lo cierto es que ningún método de valoración del cumplimiento es perfecto y este método, es valioso, porque se ha demostrado que la mayoría de los pacientes no intentan engañar a propósito a los farmacéuticos (27).

Futuras investigaciones, dada la calidad de los datos de esta cohorte y el hecho de que exista un seguimiento en el tiempo, en un par de años y a partir de nuevos cortes de este mismo registro, podrían estudiar qué factores determinan variaciones en el nivel cumplimiento en esta población de hipertensos y dislipémicos.

En conclusión, las características del paciente, la actitud del médico, la intensidad del seguimiento, el grupo de tratamiento y la simplicidad del tratamiento se relacionan con el cumplimiento en la práctica clínica diaria. Los pacientes dislipémicos tienen peor cumplimiento que los hipertensos. A pesar de obtener un cumplimiento por encima de lo publicado en la literatura, y un nivel de control por encima del promedio, todavía hay mucho espacio para mejorar.

\section{AGRADECIMIENTOS}

Al laboratorio farmacéutico Pfizer por el soporte científico, logístico y de financiación parcial prestados en esta publicación. A los diferentes profesionales de los cuatro centros de Badalona Servicios Asistenciales SA, por su constante introducción de datos en el día a día y por el rigor en su labor profesional, ya que sin su aportación, no hubiera sido posible la realización de este estudio.

\section{Bibliografía}

1. Thrall G, Lip GY, Lane D. Compliance with pharmacological therapy in hypertension: can we do better, and how? J Hum Hypertens 2004; 18 : 595-7.

2. García-Reyes Ramos M, López-Torres Hidalgo J, Ramos García E, Alcarria Rozalén A, Fernández Olano C, López Verdejo MA. Cumplimiento terapéutico en pacientes con enfermedades cardiovasculares. Med Clin (Barc) 2002; 118: 371-75.

3. Rubenfire M; Impact of Medical Subspecialty on Patient Compliance to Treatment Study Group. Safety and compliance with once-daily niacin extended-release/lovastatinas initial therapy in the Impact of Medical Subspecialty on Patient Compliance to Treatment (IMPACT) study. Am J Cardiol 2004; 94: 306-11.

4. Martín-Castillo F, Ayuga Martínez A, Perulero Escobar N, Barea Escribano N; en nombre de los miembros del estudio PRELOVAS. Adherencia al tratamiento con lovastatina en la prevención primaria de enfermedades cardiovasculares. Estudio PRELOVAS. Med Clin (Barc) 2004; 123: 692-6.
5. García Navarro MD, Orozco D, Gil V, Carratalá C, Terol C, Merino J Relación entre cumplimiento farmacológico y grado de control en pacientes con hipertensión, diabetes o dislipemia. Med Clin (Barc) 2001; 116: 141-6.

6. Insull W. The problem of compliance to colesterol altering therapy. J Intern Med 1997; 241: 317-25.

7. McCarthy R. The prize you pay for the drug not taken. Business Health 1998; 16: 27-33

8. Gascón JJ, Sánchez-Ortuno M, Llor B, Skidmore D, Saturno PJ. Treatment Compliance in Hypertension Study Group. Why hypertensive patients do not comply with the treatment: results from a qualitative study. Fam Pract 2004; 21: 125-30.

9. Wang PS, Benner JS, Glynn RJ, Winkelmayer WC, Mogun H, Avorn J. How well do patients report noncompliance with antihypertensive medications?: a comparison of self-report versus filled prescriptions. Pharmacoepidemiol Drug Saf 2004; 13: 11-9.

10. Putzer G, Roetzheim R, Ramirez AM, Sneed K, Brownlee HJ Jr, Camp- 
bell RJ. Compliance with recommendations for lipid management among patients with type 2 diabetes in an academic family practice. $\mathrm{J}$ Am Board Fam Pract 2004; 17: 101-7.

11. Piñeiro F, Gil V, Pastor R, Donis M, Torres MT, Merino J. Validez de 6 métodos indirectos para valorar el cumplimiento del tratamiento farmacológico en las dislipemias. Aten Primaria 1997; 19: 465-8.

12. Márquez Contreras E, Casado Martínez JJ, Márquez Cabeza E. Estrategias para mejorar el cumplimiento terapéutico. FMC 2001; 8: 558-73.

13. Rigueira García AI. Cumplimiento terapéutico: ¿qué conocemos de España? Aten Primaria 2001; 27: 559-68.

14. Márquez Contreras E, Casado Martínez JJ, de la Figuera Won-Vichman M, Gil Guillén V, Martell N; en representación del grupo de cumplimiento de la Sociedad Española de la HTA-LELHA. El incumplimiento terapéutico en el tratamiento de la hipertensión arterial en España. Análisis de los estudios publicados entre 1984 y 2001. Hipertensión 2002; 19: $12-6$.

15. Márquez Contreras E, Casado Martínez JJ, Corchado Albalat Y, Chaves González R, Grandío A, Losada Velasco C, Obando J, de Eugenio JM, Barrera JM. Eficacia de una intervención para mejorar el cumplimiento terapéutico en las dislipemias. Aten Primaria 2004; 33: 443-50.

16. Joint National Committee on Prevention, Detection, Evaluation and Treatment of High Blood Pressure. The sixth report of Joint National Committee on Prevention, Detection, Evaluation and Treatment of High Blood Pressure. Arch Intern Med 1997; 157: 2413-46.

17. Petrie JC, O'Brien ET, Littler WA, De Suite M; British Hypertension Society. Recommendations on blood pressure measurement. Br Med 1986: 293: 611-5.
18. Executive Summary of the Third Report of the National Cholesterol Education Program (NCEP) Expert Panel on Detection, Evaluation and Treatment on High Blood Cholesterol in Adults (Adult Treatment Panel III). JAMA 2001; 285: 2486-96.

19. Anónimo. Guidelines for ATC classification. Oslo: Nordic Collaborating Centre for Drug Statistics Methodology, 1991.

20. Lamberts H, Wood M. Clasificación Internacional de la Atención Primaria CIAP-2. Clasificación de razones de consulta. Barcelona, Masson/SG; 1990.

21. Friedewald WT, Levy RJ, Frederickson DS. Estimation of the concentration of low-density lipoproteins cholesterol in plasma without use of the preparative ultracentrifuge. Clin Chem 1972; 18: 499-502.

22. Avorn J, Monette J, Lacour A, et al. Persistence of use of lipid-lowering medications: a cross-national study. JAMA 1998; 279: 1458-62.

23. Caro JJ, Speckman JL, Salas M, Raggio G, Jackson JD. Effect of initial drug choice on persistence with antihypertensive therapy: the importance of actual practice data. CMAJ 1999; 160: 41-6.

24. Caro JJ, Salas M, Speckman JL, Raggio G, Jackson JD. Persistence with treatment for hypertension in actual practice. CMAJ 1999; 160: 31-7.

25. Lynch JC. Compliance with CAD Prevention Strategies. Requested submission. Pharmacy Times. Jan, 2000: 12-17

26. Cramer JA, Spilker B. Patient Compliance in Medical Practice and Clinical Trials. Raven Press, New York, 1991. 431 pages.

27. Spilker B. Methods of assessing and improving patient compliance in clinical practice. In Cramer and Spilker, eds: Patient compliance in medical practice and clinical trials. New York, Raven Press, 1991: 3756. 